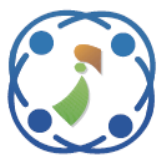

\title{
Cooperative Overlay Cognitive Radio NOMA Network with Channel Errors and Imperfect SIC
}

\author{
Nam-Soo Kim ${ }^{1 *}$ \\ ${ }^{I}$ Department of Electronic Engineering, Cheongju University, Republic of Korea \\ * Corresponding author’s Email: nskim@cju.ac.kr
}

\begin{abstract}
CR) non-orthogonal multiple access (NOMA) network which has channel estimation errors and imperfect successive interference cancellation (SIC). To further performance enhancement, spatial diversity gains can be offered by relay selection which is chosen based on channel state information (CSI) among the candidate secondary transmitters. We derive an analytical expression of the outage probability of the primary receiver (PR) and the secondary receiver (SR) as a function of the channel errors and imperfect SIC, respectively. And the analytical outage probability expressions are validated through simulations. Numerical results show that when the number of relays $(N)$ is fixed, the performance of the PR degrades as the increase of the channel estimation errors. In the case of $N=2$, to satisfy the required outage probability of $1 \times 10^{-4}, 8.2$ $\mathrm{dB}$ of the transmit SNR degradation is noticed with correlation coefficient $\rho=0.98$ in comparison with $\rho=1$. Moreover, the SNR degradation caused by the less correlation coefficient decreases as the number of relays increases. On the other hand, as further imperfect SIC, the error floor that the performance enhancement does not expect with the increase of the transmit power is noticed. The optimum power allocation which maintains the minimum outage probability of SR is realigned by the error floor.
\end{abstract}

Keywords: NOMA, Cooperative NOMA, Overlay CR, Imperfect SIC, Cognitive radio.

\section{Introduction}

According to the rapid increase of the data traffic in a cellular mobile system, the significant spectral efficiency is highly required. Recently, nonorthogonal multiple access (NOMA) has been focused as a candidate multiple access technology to utilize the efficient spectrum resources in the fifth generation (5G) mobile networks [1-4]. In NOMA system, multiple users are served at different power levels and simultaneously transmitted using the same time block. Thus, NOMA provides high throughput, high power efficiency, and low latency $[5,6]$. In addition, cooperative NOMA networks using a user relay or a dedicated relay improve communication reliability and extend cell coverage [7-10].

More recently, NOMA system with cognitive radio (CR) has been studied to achieve further spectral efficiency by sharing the licensed spectrum of a primary system. There are three representative CR methods; interweave, underlay, overlay CRs [11, 12]. When no spectrum is occupied from the licensed system, a secondary system can transmit using interweave CR. In underlay $\mathrm{CR}$, the primary and secondary system can transmit their information simultaneously under the condition of the interference on the primary network is less than the threshold level. T. Chu et al. have analyzed the performance of a cooperative NOMA system in underlay $\mathrm{CR}$ with a dedicated relay [13]. Also Y. Liu et al. have studied NOMA in large-scale underlay CR [14]. The outage probability of a user relaying cooperative NOMA with partial channel state information (CSI) in underlay CR has investigated in [15].

Meanwhile, in overlay $\mathrm{CR}$, the primary transmitter (PT) transmits, and the secondary network provides relaying the transmitted signal to the primary receiver (PR). Especially, when the PR 
is in deep fading or does not have a direct path from the PT, the PR can receive a strong signal via a relay, which contributes to lower outage probability. L. Lv et al. have considered a unicast/multicast transmissions in a NOMA system with overlay CR $[16,17]$.

In most published overlay NOMA studies assumed the perfect CSI and perfect successive interference cancellation (SIC). However, in realistic fading channels, the imperfect CSI and SIC assumption is more plausible $[18,19]$.

Motivated by the above studies, we proposed an overlay cooperative CR NOMA network model with channel errors and imperfect SIC. And we analyzed the performance of the PR and SR, respectively. To enhance the receiving performance of the PR offered by the spatial diversity gain, a selected relay is applied. We analyzed the performance degradation of the PR caused by the channel errors during the relay selection process, and derived analytical expression as a function of a correlation coefficient between the actual and the estimated channels.

Also, the impact of the residual interference components from the PT, that causes imperfect SIC, to the SR investigated. The analytical outage probability expressions have been validated through simulations.

The rest of the paper is organized as follows. In Section II, the system model of the proposed cooperative overlay CR NOMA (CON) network is described. Also the relay selection rule and the transmission protocols are presented. The outage probabilities of the PR and SR have derived analytically in Section III. In Section IV, numerical results from the analysis are presented and discussed to illustrate the impact of the network parameters on the performance of the proposed system. The results are compared with the simulation which conform the analysis. Finally, Section V provides a summary and conclusions of the paper.

\section{System model}

Fig.1 shows the proposed CON network model, where $P$ and $Q$ represent the PT and PR of the primary network, respectively. We assume NOMA is a secondary system.

In the overlay $\mathrm{CR}$ network, the secondary system provides relaying the transmitted signal from the PT to the PR. Hence, the STs of the NOMA system, which is denoted $S_{i}(i=1,2, \ldots, N)$ in Fig.1, act as relays (NOMA relays). We assume that the relays are close to each other, hence, forming a

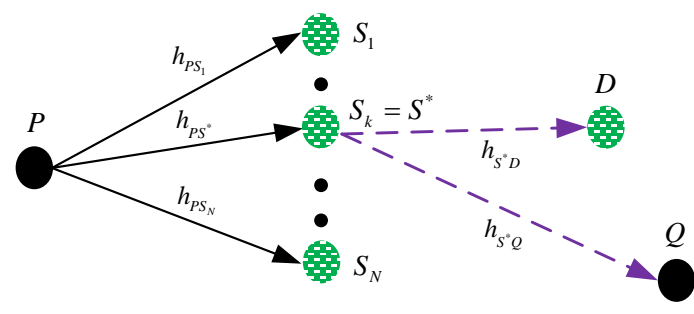

$\longrightarrow$ Phase $1 \quad-----\rightarrow$ Phase 2
P: Primary system $\quad$ 器 $D:$ Secondary (NOMA) system

Figure. 1 Proposed CON network model

cluster in Fig.1. Therefore, the distances of $P-S_{i}$, $S_{i}-Q$, and $S_{i}-D$ paths are assumed identical, respectively (i.e., $\quad d_{P S_{i}}=d_{P S^{*}}=d_{P S} \quad$, $\left.d_{S_{i} Q}=d_{S^{*} Q}=d_{S Q}, d_{S_{i} D}=d_{S^{*} D}=d_{S D}\right)$. And $D$ denotes the SR of the secondary system. Also, it is assumed the direct path between $P$ and $Q$ does not exist due to obstacles. The communication between $P$ and $Q$ can be made by introducing a NOMA relay, which ensures that the PR and SR can be served simultaneously, while sharing the licensed spectrum of the primary system.

The transmit protocol of the CON network consists of two phases. In phase1, the PT transmits messages, and $N$ NOMA relays listen. In phase 2, the selected NOMA relay $\left(S^{*}\right)$, which is a decodeand-forward relay, transmits the multiplexed messages to the PR and SR with different power levels. To improve the receiving performance of the $\mathrm{PR}$, the more power is allocated for the PR than the SR. The detailed transmit protocol is as follows.

- Phase 1 ( $P$ transmits)

In phase $1, P$ transmits and $S_{i}$ listen. The transmit signal of $P$ is $\sqrt{P_{P}} x_{P}$ where $P_{P}$ is the transmit power of $P, x_{P}$ is the message for $Q$, and the average of $E\left(\left|x_{P}\right|^{2}\right)=1$.

The received signal at $s_{i}$ can be written by

$$
y_{S_{i}}=\sqrt{P_{P S_{i}}} h_{P S_{i}} x_{P}+n_{S_{i}}
$$

where $h_{P S_{i}}$ is the channel gain of $P-S_{i}$ path, which has complex Gaussian distribution with zero mean and unit variance, $h_{P S_{i}} \sim C N(0,1)$. The received power of $P_{P S_{i}}$ is $P_{P S_{i}}=P_{P} d_{P S_{i}}^{-n}$ where $d_{P S_{i}}$ denotes the distance of $P-S_{i}$ path, and $n$ is the path loss exponent. $n_{S_{i}}$ denotes complex Gaussian noise of 
$S_{i}$ with zero mean and variance of $N_{0}, n_{S_{i}} \sim$ CN $\left(0, N_{0}\right)$.

We assumed decode-and-forward relay. The signal-to-interference plus noise ratio (SINR) of $S_{i}$ for decoding $x_{P}$ can be written by

$$
\gamma_{S_{i}}^{x_{P}}=\rho_{S_{i}}\left|h_{P S_{i}}\right|^{2}
$$

where $\rho_{S_{i}}$ is the received signal-to-noise ratio $(\mathrm{SNR})$ of $S_{i}, \rho_{S_{i}}=P_{P S_{i}} / N_{0}$.

\section{- Phase 2 (Selected relay transmits)}

In phase 2 , the selected relay transmits the messages to $Q$ and $D$, simultaneously. Before transmission, a relay is selected by the relay selection rule. There are two representative relay selection rules, max-min and max relay selection rules [20]. Max-min relay selection has better performance than max selection, however, requires global channel state information (CSI). On the other hands, max selection has less performance, but requires partial CSI, which gives less overhead to the system and less latency. In this paper, we adapt the max relay selection among the candidate relays that successfully decode the message from $P$. Let the successfully decoded relay set (decoding subset) $C$ of $N$ relays, $(|C|=I, 0 \leq I \leq N)$. In order to enhance the performance of the PR, the relay which has the maximum channel power gain of $S_{m}-Q$ path ( $S_{m} \in C, 0 \leq m \leq I$ ) among the candidate relays is selected. The index of the selected relay is given by

$$
k=\max _{S_{m} \in C}\left|h_{S_{m} Q}\right|^{2}
$$

where $\left|h_{S_{m} Q}\right|^{2}$ denotes channel power gain of $S_{m}-Q$ path. For the easy distinction, we denote the selected relay $S_{k}$ to $S^{*}$.

On the other hands, the PR sends a pilot tone to select the best relay in Eq. (3). When the candidate relays receive the pilot signal from the $P R$, the best relay that received the maximum SNR transmits first [20]. When one of the relays transmits, the other relays are silent. However, the transmitting moment of the selected relay is different from the selection moment, there are time differences. Also, in real fading environment, the channel coefficients are continuously changing. Therefore, it is expected the channel errors between the actual and the estimated channels. The actual channel can be modeled by [21]

$$
h_{S^{*} Q}=\rho \hat{h_{S^{*} Q}}+\sqrt{1-\rho^{2} \varepsilon}
$$

where $h_{S^{*} Q}$ is the actual channel coefficient of $S^{*}-Q$ path at the transmitting moment, $\hat{S_{S^{*} Q}}$ is the estimated channel coefficient at the selection moment, $\hat{h_{S}{ }^{*} Q} \sim C N(0,1) . \rho$ denotes the correlation coefficient between the actual and estimated coefficients with $0 \leq \rho \leq 1 . \varepsilon$ is the error which is independent to $\hat{S^{*} Q}$, and $\varepsilon \sim C N(0,1)$.

The selected relay decodes the message from $P$, and transmits the multiplexed signals of $\sqrt{P_{S} \alpha_{P}} x_{P}+\sqrt{P_{S} \alpha_{S}} x_{S}$, where $\alpha_{P}$ and $\alpha_{S}$ are the power allocation coefficient for $Q$ and $D$, respectively. For the better performance, more power is allocated to the PR, Q , $\alpha_{P}>\alpha_{S}$ with $\alpha_{P}+\alpha_{S}=1 . P_{S^{*}}$ is the transmit power of the selected relay. $x_{S}$ is the message for $D$, and $E\left(\left|x_{S}\right|^{2}\right)=1$.

Then, the received signal at $Q$ from the selected relay can be written by

$$
y_{Q}=\sqrt{P_{S^{*} Q^{\alpha_{P}}}} h_{S^{*} Q^{x_{P}}}+\sqrt{P_{S^{*} Q} \alpha_{S}} h_{S^{*} Q^{x_{S}}}+n_{Q}
$$

where $P_{S^{*} Q}$ is the received power, $P_{S^{*} Q}=P_{S^{*}} d_{S^{*} Q}^{-n}$. And $n_{Q}$ is the noise of $Q, n_{Q} \sim C N\left(0, N_{0}\right) . h_{S^{*} Q}$ is the channel gain of $S^{*}-Q$ path, $h_{S^{*} Q} \sim C N(0,1)$. The SINR (Signal-to-interference plus noise ratio) for decoding $x_{P}$ at $Q$ can be given by

$$
\gamma_{Q}^{x_{P}}=\frac{\alpha_{P}\left|h_{S^{*} Q}\right|^{2}}{\alpha_{S}\left|h_{S^{*} Q}\right|^{2}+1 / \rho_{Q}}
$$

where $\rho_{Q}$ is the received signal-to-noise ratio of $Q$, $\rho_{Q}=P_{S^{*} Q} / N_{0}$. The received power of $P_{S^{*} Q}$ is $P_{S^{*} Q}=P_{S^{*}} d_{S^{*}}^{-n}$ where $d_{S^{*} Q}$ denotes the distance of $S^{*}-Q$ path.

Meanwhile, the received signal at $D$ which is a secondary NOMA receiver can be written as

$$
y_{D}=\sqrt{P_{S^{*} D} \alpha_{P}} h_{S^{*} D^{X_{P}}}+\sqrt{P_{S^{*} D} \alpha_{S}} h_{S^{*} D} x_{S}+n_{D}
$$

where $h_{S^{*} D}$ is the channel gain of $S^{*}-D$ path, $h_{S^{*} D}$ $\sim C N(0,1) . n_{D}$ is the noise of $D$, and $n_{D} \sim C N\left(0, N_{0}\right)$. The received power of $P_{S^{*} D}$ is $P_{S^{*} D}=P_{S^{*}} d_{S^{*} D}^{-n}$ where 
$d_{S^{*} D}$ denotes the distance of $S^{*}-D$ path. The SINR for decoding $x_{P}$ at $D$ becomes

$$
\gamma_{D}^{x_{P}}=\frac{\alpha_{P}\left|h_{S^{*} D}\right|^{2}}{\alpha_{S}\left|h_{S^{*} D}\right|^{2}+1 / \rho_{D}} .
$$

where $\rho_{D}$ is the received signal-to-noise ratio of $D$, $\rho_{D}=P_{S^{*} D} / N_{0}$.

Generally, a NOMA receiver removes the multiplexed interference components using SIC. However, the perfect SIC is difficult and the residual interference components exist in realistic severe fading environments. After imperfect SIC, the SINR for decoding $x_{S}$ at $D$ can be written by $[18,19]$

$$
\gamma_{D}^{x_{S}}=\frac{P_{S^{*} D} \alpha_{S}\left|h_{S^{*} D}\right|^{2}}{P_{S^{*} D} \alpha_{P}\left|g_{S^{*} D}\right|^{2}+N_{0}}
$$

where $g_{S^{*} D} \sim C N(0, \beta), \quad \beta$ is the remaining interference coefficient which denotes SIC errors, and $0 \leq \beta \leq 1 . \beta=1$ denotes no SIC is applied, and $\beta=0$ means the perfect SIC case. The interference term plus noise in Eq. (9) can be approximated to another complex Gaussian distribution, hence, Eq. (9) can be written by [22]

$$
\gamma_{D}^{x_{S}} \approx \frac{P_{S^{*} D} \alpha_{S}\left|h_{S^{*} D}\right|^{2}}{N_{t}}
$$

where $N_{t}=P_{S^{*} D} \alpha_{P} \beta+N_{0}$.

\section{Outage analysis}

Since the messages to the PR and SR are different, this section derives the outage probability of the PR and SR separately.

\subsection{Outage probability of the primary receiver}

The outage happens when the transmission of the selected relay, which is selected among the successfully decoded relays, is failed. The outage probability of $Q$ can be written by

$$
P_{O, Q}=\sum_{l=0}^{N} \operatorname{Pr}\left(\gamma_{Q}^{x_{P}}<\Gamma_{P}|C|=l\right) \operatorname{Pr}(|C|=l)
$$

where $C$ denotes decoding subset and $|C|$ denotes the number of the successful relays (Cardinality) as International Journal of Intelligent Engineering and Systems, Vol.12, No.5, 2019 mentioned earlier. $\operatorname{Pr}\left(\gamma_{Q}^{x_{P}}<\Gamma_{P}|| C \mid=1\right)$ is the conditional outage probability of $S^{*}-Q$ path; when the received SINR at $Q$ is below the threshold with a given condition of $|C|=I$, an outage happens. And $\Gamma_{P}=2^{2 R_{P}}-1$ which is the threshold, where $R_{P}$ is the required spectral efficiency of $Q$ in $[\mathrm{bps} / \mathrm{Hz}]$.

From Eq. (6), the conditional outage probability of $S^{*}-Q$ path, $\operatorname{Pr}\left(\gamma_{Q}^{X_{P}}<\Gamma_{P}|| C \mid=l\right)$ can be written by

$$
\begin{gathered}
\operatorname{Pr}\left(\left|h_{S^{*} Q}\right|^{2}<\frac{\Gamma_{P}}{\rho_{Q}\left(\alpha_{P}-\alpha_{S} \Gamma_{P}\right)}|| C \mid=l\right) \\
\quad=F_{H_{S^{*} Q}}(\eta|| C \mid=l), \quad \Gamma_{P}<\frac{\alpha_{P}}{\alpha_{S}}
\end{gathered}
$$

where $\eta=\Gamma_{P} / \rho_{Q}\left(\alpha_{P}-\alpha_{S} \Gamma_{P}\right)$ and $H_{S^{*} Q}=\left|h_{S^{*} Q}\right|^{2}$. The cumulative distribution function (CDF) of $H_{S^{*} Q}$ can be derived by integrating the probability density function (PDF). The PDF can be written by

$$
f_{H_{S^{*} Q}}(z)=\int_{0}^{\infty} f_{H_{S^{*} Q} \mid \hat{H}_{S^{*} Q}}(z \mid x)_{\hat{H}_{S^{*} Q}}(x) d x
$$

where $\hat{H}_{S^{*} Q}$ is the estimate of $H_{S^{*} Q}$. The conditional $\mathrm{PDF}$ is given as [23]

$$
f_{H_{S^{*} Q} \mid \hat{H}_{S^{*} Q}}(z \mid x)=\frac{1}{1-\rho^{2}} e^{-\frac{z+\rho^{2} x}{1-\rho^{2}}} I_{0}\left(\frac{2 \rho \sqrt{z x}}{1-\rho^{2}}\right)
$$

where $I_{0}(\bullet)$ stands for the zero-order modified Bessel function of the first kind. By inserting Eq. (14) and after rearrangement, Eq. (13) can be rewritten by [10]

$$
f_{H_{S^{*} Q}}(z)=\sum_{n=1}^{l}\left(\begin{array}{l}
I \\
n
\end{array}\right)(-1)^{n-1} \frac{n}{\rho^{2}+\left(1-\rho^{2}\right) n} e^{-\frac{z}{1-\rho^{2}}\left\{1-\frac{\rho^{2}}{\rho^{2}+\left(1-\rho^{2}\right)}\right\}} .
$$

Therefore, we can obtain the CDF by integrating Eq. (15), which is the conditional probability of Eq. (12) is given by

$$
\begin{aligned}
F_{H_{s^{*} Q}}(\eta|C|=I) & =\int_{0}^{\eta} f_{H_{s^{*} Q}}(z) d z \\
& =\sum_{n=1}^{l}\left(\begin{array}{l}
l \\
n
\end{array}\right)(-1)^{n-1} \frac{n}{\rho^{2}+\left(1-\rho^{2}\right) n} \Psi_{a}(\eta)
\end{aligned}
$$


where $a=\frac{1}{1-\rho^{2}}\left\{1-\frac{\rho^{2}}{\rho^{2}+\left(1-\rho^{2}\right) n}\right\}$.

and

The second probability of Eq. (11), $\operatorname{Pr}(|c|=I)$ can be written by

$$
\operatorname{Pr}(|C|=I)=\left(\begin{array}{l}
N \\
I
\end{array}\right)\left[\operatorname{Pr}\left(\gamma_{S^{*}}^{x_{P}} \geq \Gamma_{P}\right)\right]^{\prime}\left[1-\operatorname{Pr}\left(\gamma_{S^{*}}^{x_{P}} \geq \Gamma_{P}\right)\right]^{N-I} .
$$

The first probability of Eq. (17) can be obtained from Eq. (2),

$$
\operatorname{Pr}\left(\gamma_{S^{*}}^{x_{P}} \geq \Gamma_{P}\right)=\operatorname{Pr}\left(\left|h_{P S^{*}}\right|^{2} \geq \frac{\Gamma_{P}}{\rho_{S}}\right)=e^{-\frac{\Gamma_{P}}{\rho_{S}}}
$$

The probability of $\operatorname{Pr}(|C|=l)$ in Eq. (17) can be obtained from Eq. (18). Consequently, the outage probability of $Q$ which is given in Eq. (11) can be obtained from Eqs. (16) and (17).

\subsection{Outage probability of the secondary receiver}

The outage probability of $D$ can be denoted by multiplying the success probability of $P-S^{*}$ path $\left(P_{\text {succ }, P S^{*}}\right)$ by the outage probability of $S^{*}-D$ path $\left(P_{O, S^{*} D}\right)$ plus the outage probability of $P-S^{*}$ path, which is given by

$$
P_{O, D}=P_{\text {SUCC }, P S^{*}} \times P_{O, S D}+\left(1-P_{\text {SUCC }, P S^{*}}\right)
$$

where $P_{\text {succ, } P S^{*}}$ is

$$
P_{\text {SUCC }, P S^{*}}=\operatorname{Pr}\left(\gamma_{S^{*}}^{x_{P}} \geq \Gamma_{P}\right),
$$

and it is given in Eq. (18).

Since $D$ is the NOMA receiver, the outage probability of $P_{O, S^{*} D}$ in Eq. (19) is given by [8]

$$
P_{O, S^{*} D}=\operatorname{Pr}\left(\gamma_{D}^{x_{P}}<\Gamma_{P}\right)+\operatorname{Pr}\left(\gamma_{D}^{x_{P}} \geq \Gamma_{P}, \gamma_{D}^{x_{S}}<\Gamma_{S}\right)
$$

where $\Gamma_{S}=2^{R_{S}}-1$, and $R_{S}$ is the required spectral efficiency of the $\mathrm{SR}$ in $[\mathrm{bps} / \mathrm{Hz}]$. By replacing Eq. (8) and Eq. (10) into Eq. (21), and after rearrangement the outage probability becomes

$$
\begin{gathered}
P_{O, S^{*} D}=\operatorname{Pr}\left(\left|h_{S}{ }^{*}\right|^{2}<\max \left\{\frac{\Gamma_{P}}{\left(\alpha_{P}-\Gamma_{P} \alpha_{S}\right)_{\rho_{D}}}, \frac{\Gamma_{S}}{\alpha_{S}}\left(\alpha_{P} \beta+\frac{1}{\rho_{D}}\right)\right\}\right), \\
\Gamma_{P}<\frac{\alpha_{P}}{\alpha_{S}} .
\end{gathered}
$$

The selected relay, as explained in Eq. (3), is selected based on the channel gain of $S_{m}-Q$ paths, not $S_{m}-D$ paths. Therefore, the selected relay becomes a best relay only for the PR. To the point of the SR, the selected relay does not guarantee the maximum channel gain of $S_{m}-D$ path. It is one of the transmitters in Rayleigh fading channel. By applying the exponential pdf of $\left|h_{S^{*} D}\right|^{2}$, Eq. (22) can be written by

$$
\begin{aligned}
P_{O, S^{*} D} & =1-\exp \left(-\max \left\{\frac{\Gamma_{P}}{\left(\alpha_{P}-\Gamma_{P} \alpha_{S}\right) \rho_{D}}, \frac{\Gamma_{S}}{\alpha_{S}}\left(\alpha_{P} \beta+\frac{1}{\rho_{D}}\right)\right\}\right), \\
\Gamma_{P} & <\frac{\alpha_{P}}{\alpha_{S}} .
\end{aligned}
$$

\section{Numerical examples}

We assume the system parameters for the outage analysis are $R_{P}=R_{S}=1[\mathrm{bps} / \mathrm{Hz}], n=3$. Also, it is assumed the normalized distances $d_{P S^{*}}=d_{S^{*} Q}=0.5$ which are normalized to the distances between the primary nodes, the PS and PR. The transmit powers of the PS and the selected relay are assumed identical.

Fig.2 shows the outage probability of the PR with different correlation coefficients, where “*” denotes the simulation results. Analytical results are coincide with that of the simulation which confirms the accuracy of the analysis. It is shown that the performance improves with the number of relays, which is the exploits of the spatial diversity.

As expected, when the number of relays is fixed, the outage probability increases as the correlation between the actual and the estimated channel decreases. In the case of $P_{P} / N_{0}=15 \mathrm{~dB}$ with $N=5$, the outage probability increases from $1.5 \times 10^{-6}$ to $7.7 \times 10^{-5}$ for $\rho=1$ and $\rho=0.98$, respectively. That is, when the number of relays is fixed, the transmit SNR $\left(P_{P} / N_{0}\right)$ needs to be increased with the decrease of the correlation coefficient to satisfy the required outage probability. In the case of $N=2$, the transmit SNR which satisfy the required outage probability of $1 \times 10^{-4}$ is $23.4 \mathrm{~dB}$ and $31.6 \mathrm{~dB}$ with $\rho=1$ and $\rho=0.98$, respectively; it shows $8.2 \mathrm{~dB}$ SNR degradation. Moreover, the SNR degradation caused by the less correlation decreases as the number of relays increases. 


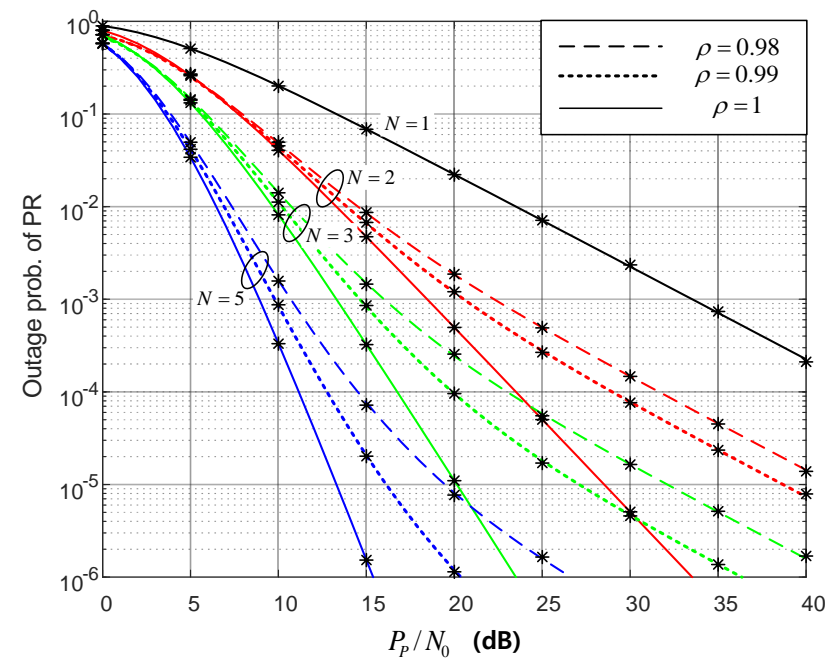

Figure.2 Outage probability of the primary receiver with different correlation coefficients $\left(R_{P}=R_{S}=1, P_{P}=P_{S^{*}}\right.$,

$$
d_{P S^{*}}=d_{S^{*} Q}=0.5, n=3, \alpha_{P}=0.8, \alpha_{S}=0.2 \text { ) }
$$

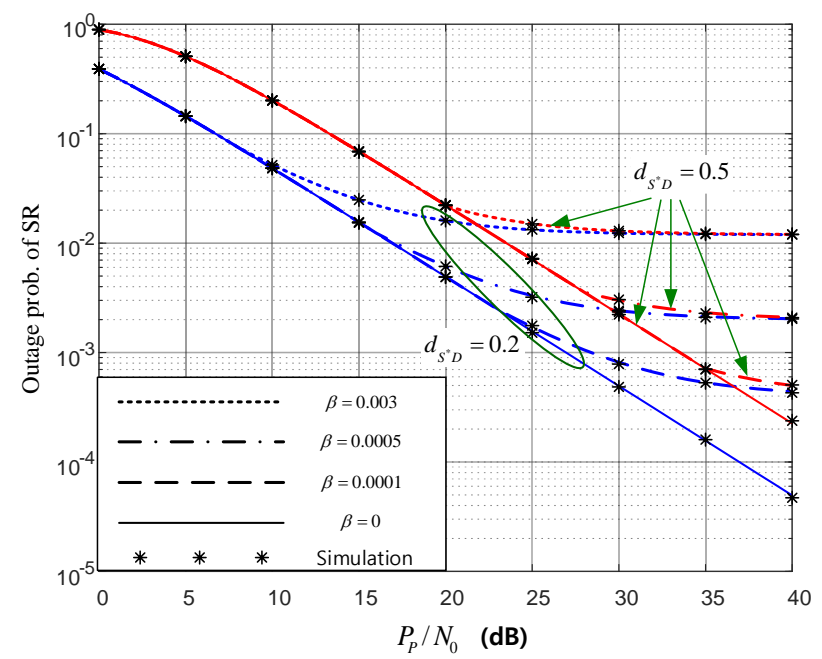

Figure. 3 Outage probability of the secondary receiver with different SIC errors $\left(R_{P}=R_{S}=1, d_{P S} * 0.5\right.$,

$$
\left.P_{P}=P_{S^{*}}, n=3, \alpha_{P}=0.8, \alpha_{S}=0.2\right)
$$

Fig. 3 shows the outage probability of the SR with different SIC errors at $d_{S^{*} D}=0.2$ and 0.5. It is noticed that the performance of the SR is very sensitive to the SIC errors. Especially, the error floor that the performance improvement does not expect with the increase of the transmit SNR is noticed. Therefore, the perfect SIC is very important for the performance enhancement.

Generally, the more power is allocated to the far user for the performance improvement in a NOMA system. In this paper, we allocate more power to the PR which does not have a direct path from the PT this is the reason why we introduce the NOMA relay. Since the total transmit power is fixed, the less power is allocated to the SR. Therefore, it is

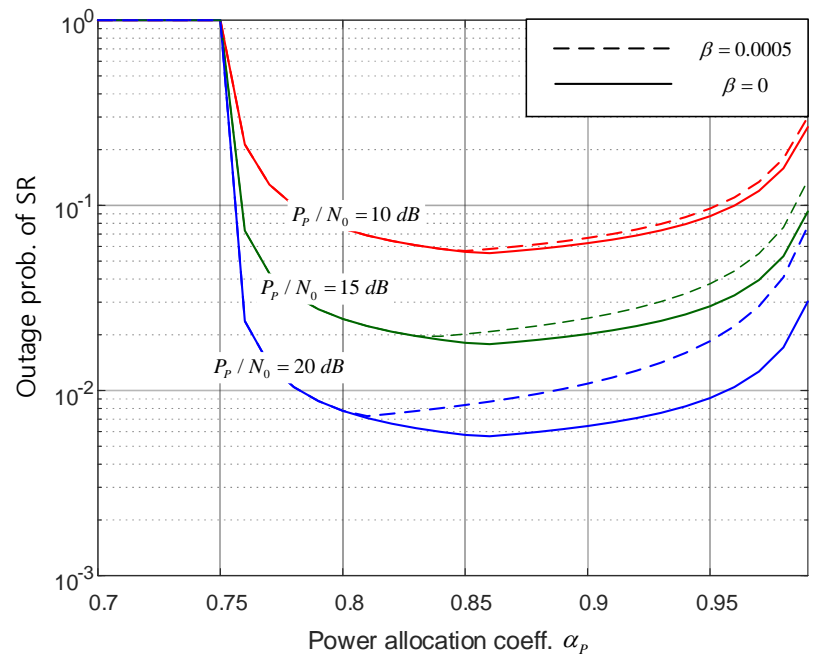

Figure.4 Outage probability of the secondary receiver vs. power allocation coefficients $\left(R_{P}=R_{S}=1, d_{P S^{*}}=0.5\right.$,

$$
\left.d_{S^{*} D}=0.3, P_{P}=P_{S^{*}}, n=3\right)
$$

meaningful to consider the influence of the power allocation to the performance of the SR, which is a NOMA user.

In Fig.4, the outage probability of the SR $\left(P_{O, D}\right)$ versus power allocation coefficient, which shows convection curbs. It means that there is an optimum power allocation to minimize the outage probability of the SR. Without SIC errors $(\beta=0)$, the optimal power allocation coefficient which maintains the minimum outage probability is fixed to $\alpha_{P}=0.86$, irrespective of the transmit SNR. With the SIC errors of $\beta=0.0005$, it is noticed that the optimum coefficient decreases to $0.85,0.84$, and 0.81 as the transmit SNR increases to $10,15,20 \mathrm{~dB}$, respectively. It is interpreted that the error floor caused by SIC error shifts the optimum power allocation coefficient.

\section{Conclusions}

We have proposed a cooperative overlay CR NOMA network and analyzed the performance of the PR and SR with channel errors and SIC errors.

The analytical expressions of the outage probability of the PR and SR have been derived in closed form and conformed through simulation.

The numerical results illustrated that when the number of relays is fixed, the performance of the PR degrades as the increase of the channel estimation errors. In the case of $N=2$, to satisfy the required outage probability of $1 \times 10^{-4}, 8.2 \mathrm{~dB}$ of the transmit SNR degradation is noticed with $\rho=0.98$ in comparison with $\rho=1$. As the number of relays decreases, the more performance degradation is noticed with the channel estimation errors. 
On the other hand, the performance of the SR, which is NOMA user, shows very sensitive to the SIC errors. When the imperfect SIC increases, the error floor that the performance enhancement does not expect with the increase of the transmit power is noticed. The residual interference components prevent the performance enhancement. Accordingly, the SIC error must be controlled carefully to improve the performance of the SR.

Though the performance of the PR improves with the increase of the power allocation to the PR, that of the SR does not monotonically increase or decrease. It has an optimal power allocation. When there are no SIC errors, the optimal coefficient which minimizes the outage probability is fixed irrespective of the transmit SNR. When the SIC error exists, it is noticed that the optimum power allocation coefficient is shifting towards smaller value as the transmit SNR increases. It is interpreted that the error floor incurs these phenomena.

The further research will be focused on the performance enhancement of the SR by considering an efficient $\mathrm{CR}$ network configuration.

\section{References}

[1] Y. Saito, Y. Kishiyama, A. Benjebbour, T. Nakamura, A. Li, and K. Higuchi, "Nonorthogonal Multiple Access (NOMA) for Cellular Future Radio Access", In: Proc. of Vehicular Technology Conference, pp.1-5, 2013.

[2] Z. Ding, Z. Yang, P. Fan, and H. V. Poor, "On the Performance of Non-orthogonal Multiple Access in 5G Systems with Randomly Deployed Users", IEEE Signal Processing Letters, Vol.21, No.12, pp. 1501-1505, 2014.

[3] Z. Ding, M. Peng, and H. V. Poor, "Cooperative Non-orthogonal Multiple Access in 5G Systems", IEEE Communications Letters, Vol.19. No.8, pp.1462-1465, 2015.

[4] L. Dai, B. Wang, Y. Yuan, S. Han, C.-1. I, and Z. Wang, "Non-orthogonal Multiple Access for 5G: Solutions, Challenges, Opportunities, and Future Research Trends", IEEE Communications Magazine, Vol.53, No.9, pp.74-81, 2015.

[5] S. M. R. Islam, N. Avazov, O. A. Dobre, and K. S. Kwak, "Power-domain Non-orthogonal Multiple Access (NOMA)) in 5G Systems: Potentials and Challenges", IEEE Communications Surveys \& Tutorials, Vol. 19, No.2, pp. 721-742, 2016.

[6] H. Liu, Z. Ding, K. J. Kim, K. S. Kwak, and H. V. Poor, "Decode-and-forward Relaying for
Cooperative NOMA Systems with Direct link", IEEE Transactions on Wireless Communications, Vol.17, No.12, pp.8077-8093, 2018.

[7] Z. Ding, H. Dai, and H. V. Poor, "Relay Selection for Cooperative NOMA", IEEE Wireless Communications Letters, Vol.5, No.4, pp.416-419, 2016.

[8] Y. Liu, Z. Ding, M. Elkashlan, and H. V. Poor, "Cooperative Non-orthogonal Multiple Access with Simultaneous Wireless Information and Power Transfer", IEEE Journal on Selected Areas in Communications, Vol.34, No.4, pp.938-953, 2016.

[9] N.-S. Kim, "Performance of Max-min User Relay Cooperative NOMA Systems with Imperfect CSI", International Journal of Intelligent Engineering and Systems, Vol.11, No.6, pp.148-155, 2018.

[10] N.-S. Kim, "Performance of User Relay Cooperative NOMA System with Partial and Imperfect CSI", International Journal of Engineering and Technology, Vol.7, No.4, pp.4462-4467, 2019.

[11] L. Lv, J. Chen, Q. Ni, Z. Ding, and H. Jiang, "Cognitive Non-orthogonal Multiple Access with Cooperative Relaying: a New Wireless Frontier for 5G Spectrum Sharing", IEEE Communications Magazine, Vol.56, No.4, pp.188-195, 2018.

[12] A. Goldsmith, S. A. Jafar, I. Maric, and S. Srinivasa, "Breaking Spectrum Gridlock with Cognitive Radios: an Information Theoretic Perspective", In: Proc. of the IEEE, Vol.97, No.5, pp.894-914, 2009.

[13] T.M.C. Chu and H.-J. Zepernick, "Downlink Outage Analysis for Cognitive Cooperative Radio Networks Employing Non-orthogonal Multiple Access", In: Proc. of International Conference on Communications and Electronics, pp.27-32, 2018.

[14] Y. Liu and Z. Ding, "Nonorthogonal Multiple Access in Largescale Cognitive Radio Networks", IEEE Transactions on Vehicular Technology, Vol.65, No.12, pp.10152-10157, 2016.

[15] N.-S. Kim, "Performance of User Relaying Cooperative NOMA System Underlay Cognitive Radio Networks with Partial CSI", International Journal of Intelligent Engineering and Systems, Vol.12, No.2, pp.287-296, 2019.

[16] L. Lv, J. Chen, and Q. Ni, "Cooperative Nonorthogonal Multiple Access in Cognitive Radio", IEEE Communications Letters, Vol.20, No.10, pp.2059-2062, 2016. 
[17] L. Lv, L. Yang, H. Jiang, T. Luan, and J. Chen, "When NOMA Meets Multiuser Cognitive Radio: Opportunistic Cooperation and User Scheduling", IEEE Transactions on Vehicular Technology, Vol. 67, No. 7, pp. 6679-6684, 2018.

[18] M. F. Kader, M. B. Shahab, and S. Y. Shin, "Exploiting Non-orthogonal Multiple Access in Cooperative Relay Sharing", IEEE Communications Letters, Vol. 21, No. 5, pp. 1159-1162, 2017.

[19] C. Zhong and Z. Zhang, "Non-orthogonal Multiple Access with Cooperative Full-duplex Relaying", IEEE Communications Letters, Vol. 20, No. 12, pp. 2478-2481, 2016.

[20] A. Bletsas, H. Shin, and M. Z. Win, "Cooperative Communications with Outageoptimal Opportunistic Relaying", IEEE Transactions on Wireless Communications, Vol.6, No.9, pp.3450-3460, 2007.

[21] V. Sharma, K. Premkumar, and R. N. Swamy, "Exponential Diversity Achieving Spatiotemporal Power Allocation Scheme for Fading Channels", IEEE Trans. on Information Theory, Vol. 54, No. 1, pp.188-208, 2008.

[22] Md. F. Kader, M. B. Shahab, and S. Y. Shin, "Exploiting Non-orthogonal Multiple Access in Cooperative Relay Sharing", IEEE Communications Letters, Vol. 21, No. 5, pp. 1159-1162, 2017.

[23] L. Vicario and C. Anton-Haro, "Analytical Assessment of Multi-user vs. Spatial Diversity Trade-offs with Delayed Channel State Information", IEEE Communications Letters, Vol.10, No.8, pp.588-590, 2006. 